\title{
Late-onset postoperative Mycobacterium haemophilum endophthalmitis masquerading as inflammatory uveitis: a case report
}

Warinyupa Pinitpuwadol, Sucheera Sarunket, Sutasinee Boonsopon, Nattaporn Tesavibul and Pitipol Choopong (i)

\begin{abstract}
Background: Although atypical mycobacteria had been increasingly found in various ocular infections in the past decades, a slow-growing Mycobacterium haemophilum (M. haemophilum) was scarcely reported. Similar to tuberculous infection, the presentation can masquerade as low-grade granulomatous intraocular inflammation with partial response to corticosteroids. Besides, the special requirements for culture make this pathogen difficult to diagnose. The study aims to report the clinical presentation and notify the awareness of NTM endophthalmitis among clinicians. This is the first case report of late-onset, postoperative M. haemophilum endophthalmitis in the literature.
\end{abstract}

Case presentation: A 66-year-old man with non-insulin-dependent diabetes mellitus (NIDDM) manifested chronic granulomatous inflammation in the left eye after multiple glaucoma surgeries. With a diagnosis of noninfectious panuveitis, he was treated with systemic corticosteroids. The inflammation initially responded to therapy although it subsequently worsened and became purulent endophthalmitis. The vitreous cultures grew M. haemophilum. Intraocular and systemic antimicrobial treatments were administered early, but the patient eventually turned blind.

Conclusions: $M$. haemophilum endophthalmitis is a rare but serious intraocular complication leading to loss of vision or eyeball. Awareness of atypical mycobacterial infections is necessary especially in patients with impaired immune function, previous intraocular surgery, and corticosteroid resistance. Proper laboratory investigations and treatments should be performed. However, due to the rarity of the disease, the development of guidelines for its investigation and therapy is still challenging.

Keywords: Atypical mycobacteria, Non-tuberculous mycobacteria, Endophthalmitis, Mycobacterium haemophilum

\section{Background}

Non-tuberculous mycobacteria (NTM), or atypical mycobacteria, are described as Mycobacterium species other than M. tuberculosis. One of these, M. haemophilum, is a member of the slow-growing NTM which is nonphotochromogenic and grows over two-four weeks [1]. This aerobic, non-spore-forming, gram-positive bacterium is found in the general environment; some studies have suggested that water is the main reservoir of M. haemophilum [2-5].

\footnotetext{
* Correspondence: pitipol.cho@mahidol.edu; pitipol@gmail.com Department of Ophthalmology, Faculty of Medicine, Siriraj Hospital, Mahidol University, 2 Wanglang Road, Bangkok Noi, Bangkok 10700, Thailand
}

M. haemophilum was first introduced as a new pathogen in 1978 in a case of Hodgkin's lymphoma presenting with chronic skin infection [6]. Later, it was reported in a variety of infections, mainly in immunocompromised hosts and children [3, 4, 7-9]. Three cases of $M$. haemophilum ocular infections have been reported in literature. Millar et al. reported an acute myeloid leukemia patient with NIDDM who developed chronic conjunctivitis and interstitial keratitis with multiple skin lesions on the face and arms [10]. Zuercher et al. reported a case of healthy child who presented with fistulous dacryocystitis and multiple cervical lymphadenopathies [11]. The only intraocular M. haemophilum infection was reported by Modi et al. in 2007; the authors described a case of 
chronic endogenous endophthalmitis with multiple skin nodules in a patient who was receiving immunosuppressive drugs after cardiac transplantation [12].

In this paper, we report a case of late-onset, postoperative endophthalmitis caused by $M$. haemophilum at the Department of Ophthalmology, Siriraj Hospital, Mahidol University, Bangkok, Thailand. Our patient manifested with an inflammatory uveitis-like presentation which partially responded to systemic corticosteroids and later became infectious endophthalmitis. He ended up with phthisis bulbi. To our knowledge, this is the first case of late-onset, postoperative endophthalmitis caused by $M$. haemophilum.

\section{Case presentation}

A 66-year-old Thai man with bilateral advanced primary open angle glaucoma (POAG) presented to our clinic with chronic panuveitis for four months. His medical conditions included hypertension, hypercholesterolemia, and non-insulin-dependent diabetes mellitus (NIDDM) with an $\mathrm{HbA1C}$ of $8.2 \%$. The patient had undergone three trabeculectomy surgeries in each eye; the last surgery having been performed in the left eye five years prior to the intraocular inflammation. Four months before presentation to our clinic, he complained of diminution of vision, pain, and redness in the left eye, which was treated as inflammatory panuveitis by an ophthalmologist at a local hospital. The treatment included topical and systemic corticosteroids. The inflammation improved during the three-month treatment period, but recurred after discontinuation of the medications. The second attempt showed minimal improvement over one month. Therefore, he stopped the medications and sought our opinion.

At our service, the patient reported blurred vision with no ocular pain. The initial examination showed $6 / 6$ bestcorrected visual acuity (BCVA) in the right eye, and a poor response to light projection (PJ) in the left eye. The intraocular pressure (IOP) measurements were $21 \mathrm{mmHg}$ in the right eye and $15 \mathrm{mmHg}$ in the left eye. Slit-lamp examination of the left eye showed three flat trabeculectomy blebs with moderate circumcorneal conjunctival injection without discharge. Several large, mutton-fat keratic precipitates (KPs) were observed. There were plasmoid aqueous and $4+$ cells in the anterior chamber (Fig. 1). A fundus examination revealed severe vitritis with a string-of-pearls appearance. The right eye was normal with three flat trabeculectomy blebs.

A moderate heterogenous vitreous echogenicity and attached retina appeared on B-scan ultrasonography. Chest radiography was normal. Laboratory results for anti-HIV, VDRL, TPHA, rheumatoid factor, antinuclear antibodies, and toxoplasma serology were all negative. Unfortunately, tests for serum lysozyme and angiotensin-converting

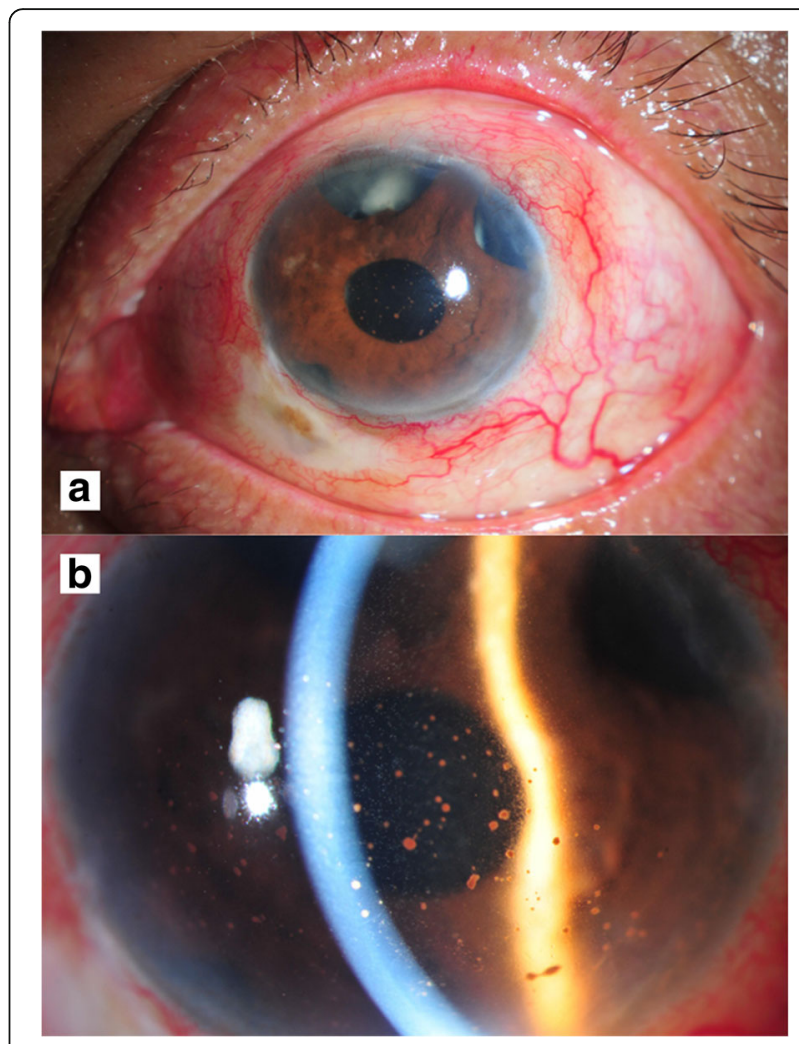

Fig. 1 Slit lamp examination of the left eye at initial presentation. a Three flat trabeculectomy blebs with moderate circumcorneal conjunctival injection (b) Plasmoid aqueous, 4+ cells, and several large mutton fat keratic precipitates in the anterior chamber

enzyme were not performed as they were not available in Thailand. A presumptive diagnosis of severe panuveitis was made although infectious causes could not be excluded. Oral prednisolone was started at a dosage of $1 \mathrm{mg} / \mathrm{kg} /$ day with caution. The inflammation subsided. The visual acuity improved to hand motion (HM), and the prednisolone was gradually tapered.

One month later, his symptoms worsened with moderate ocular pain. Intense inflammation, including $4+$ cells, iris fibrinous membrane, and hypopyon, was observed in the left eye. The inferior filtering bleb became inflamed. Due to these findings, infectious endophthalmitis became more suspicious. Aqueous and vitreous aspirations for cultures and molecular detections were performed to identify the organism, along with intravitreal injections of vancomycin $(1 \mathrm{mg} / 0.1 \mathrm{ml})$ and amikacin $(400$ $\mathrm{mcg} / 0.1 \mathrm{ml}$ ). After two unsuccessful results from aqueous and vitreous samples, pars plana vitrectomy (PPV) and iris membranectomy were performed, with additional intravitreal injections of vancomycin $(1 \mathrm{mg} / 0.1 \mathrm{ml})$ and ceftazidime $(2.25 \mathrm{mg} / 0.1 \mathrm{ml})$. The iris membrane was sent for microbial cultures and histopathological examination. The vitreous fluid was sent for direct microscopic 
examination, culture, and molecular identification of bacteria, fungi, and mycobacteria. The patient was admitted and treated with fortified vancomycin and amikacin eye drops every hour concurrent with intravenous administration of both medications (vancomycin $1 \mathrm{~g} /$ day and amikacin $750 \mathrm{mg} /$ day). Two days after PPV, vitreous staining indicated $1+$ acid-fast bacilli, but the polymerase chain reaction (PCR) was negative for mycobacteria. All other investigations for bacteria and fungi appeared negative.

According to the test results, NTM endophthalmitis was mostly suspected. The treatment was changed according to the infectious disease specialist's recommendation to intravenous imipenem (4 g/day), levofloxacin $(750 \mathrm{mg} /$ day), and amikacin $(750 \mathrm{mg} /$ day $)$ combined with levofloxacin eye drops every hour and tobramycin eye ointment before bedtime. After two weeks, the intravenous antibiotics were switched to oral doxycycline (200 mg/day), clarithromycin (1 g/day), and ciprofloxacin $(1500 \mathrm{mg} /$ day). Intermittent local injections (intravitreal, intracameral, and subconjunctival) of amikacin and imipenem were performed as an adjunctive to the systemic and topical treatments. The ocular pain and intraocular inflammation gradually improved over the treatment period. Despite the clinical improvement, the patient's visual acuity deteriorated to no light perception (NPL), and uveal tissue had prolapsed through the superotemporal scleral window of a trabeculectomy wound (Fig. 2). Enucleation of the infected eyeball was advised to prevent sympathetic ophthalmia, but the patient declined. Three months later, vitreous cultures revealed M. haemophilum in liquid medium. Unfortunately, the organism could not be identified in the solid medium even after sub-culturing from liquid to solid medium, so the antibiotic susceptibility test could not be done in this patient. The final regimen was

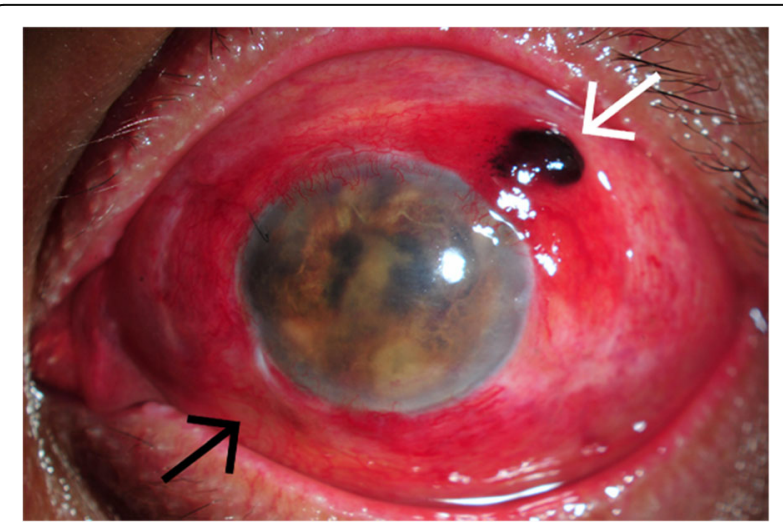

Fig. 2 Slit lamp examination of the left eye at three months after. Prolapsed uveal tissue through a superotemporal scleral window of trabeculectomy wound (white arrow) and the inflamed inferior filtering bleb (black arrow) changed to oral azithromycin (500 mg/day), doxycycline $(200 \mathrm{mg} /$ day $)$, and rifampicin $(600 \mathrm{mg} /$ day $)$ for the next 12 months. The eye eventually became phthisical although there was no sign of recurrence or systemic infection at the five-year follow-up.

\section{Discussion and conclusions}

To date, only three cases of M. haemophilum ocular infection have been published in previously reported literature [3, 10-12]. The common features were cutaneous lesions and an incomplete or impaired immune function, including young age, immunosuppressed, or uncontrolled diabetes. Cutaneous lesions were early, and were the common signs of $M$. haemophilum infection in all of the reported cases of ocular infection [3, 8, 10-12]. Therefore, ocular involvement appeared to be secondary to hematogenous spread. One of three reported cases was endophthalmitis as in our case [12]. The patient had cutaneous lesions and a gradual history of iridocyclitis before developing a suppurative, granulomatous ocular inflammation which finally required enucleation. Our patient demonstrated a similar clinical course. He had uncontrolled diabetes and developed ocular inflammation mimicking chronic granulomatous panuveitis. Subsequently, he ended up with purulent endophthalmitis and phthisis bulbi, despite the combination of intraocular and systemic antimicrobial agents.

Unlike the previously reported cases, our case was the first to demonstrate an ocular M. haemophilum infection without any signs of systemic association. The route of infection in our patient was assumed to be exogenous due to the history of multiple trabeculectomies. From previous studies, the five-year incidence of bleb-related endophthalmitis is $1.3 \%$, and the common route for late post-trabeculectomy endophthalmitis is an ingress of microorganisms through the avascular bleb [13-16]. Nevertheless, in our case, the inoculation of mycobacteria through blebs could not be concluded as there was no sign of bleb inflammation at the initial presentation. Since there was no blebitis at the beginning, the condition misled us for immune-related process and thus delayed the investigations and treatment.

$M$. haemophilum infection is difficult to diagnose for the lack of specific signs, the chronic and subtle course of disease, and the special culture requirements. This slow-growing mycobacterium usually takes up to eight weeks to grow in a medium in the temperature range of $30-32{ }^{\circ} \mathrm{C}$, which is lower than that required by other NTMs $\left(35-37^{\circ} \mathrm{C}\right)$. Specifically, it needs iron supplementation in the culture medium and this characteristic gives it the name "haemophilum." Improper culture techniques may lead to a false-negative result $[3,8,11,17,18]$. A regimen for $M$. haemophilum diagnosis includes acid-fast stain with Ziehl-Neelsen techniques and two culture 
conditions: 1) standard protocol, using Mycobacteria Growth Indicator Tube (MGIT) and Löwenstein-Jensen (LJ) medium at $35{ }^{\circ} \mathrm{C}$; and 2) M. haemophilum-specific protocol, using $\mathrm{LJ}$ medium with iron supplementation at $30{ }^{\circ} \mathrm{C}$. Molecular detection is also recommended because a PCR assay provides higher sensitivity for $M$. haemophilum detection [3]. In our case, although treatment was initiated as soon as NTM was suspected, it took three months to identify the organism.

Even though the drug susceptibility of microorganism in our case was not established, previous studies revealed that NTM was commonly susceptible to antituberculosis, fluoroquinolones, macrolides, and aminoglycosides $[3,14,20]$. Therefore, our suggested treatment is a long-term systemic combination of rifampicin, ciprofloxacin, doxycycline, and azithromycin. Additional intravitreal injection of amikacin was also recommended for NTM intraocular infection [3, 14]. However, specific guidelines for the antibiotics, the routes of administration and the duration of treatment have not been developed $[3,8,19]$.

The prognosis depends on early diagnosis, appropriate therapy, and immune status of the patient which affects immune function [20]. White et al. described that early diagnosis could reduce morbidity and mortality in bone marrow transplant recipients with $M$. haemophilum infection [21]. Fairhurst et al. emphasized the importance of early treatment which led to good outcome, especially in disseminated M. haemophilum infection [22]. Similar to the previous report, our case culminated with NPL and phthisis bulbi, despite aggressive treatments with local and systemic antimicrobial agents. This should alert the ophthalmologist to perform microorganism identification including NTM as soon as infection is suspicious.

Although rare, NTM endophthalmitis can be devastating to the eye. Unlike previous reports, our case was the first of exogenous endophthalmitis being caused by $M$. haemophilum. This case signals the need to be aware of NTM infections when face with cases of chronic-onset granulomatous intraocular inflammation, and with histories of previous intraocular intervention, immunocompromised status, and/or corticosteroid resistance. Early identification of the microorganisms and proper treatments are required to minimize the risk of losing a patient's eye. However, guidelines for investigation and therapeutic intervention are not well established.

\footnotetext{
Abbreviations

BCVA: best-corrected visual acuity; HM: hand motion; IOP: intraocular pressure; KPs: keratic precipitates; L: Löwenstein-Jensen; $M$. haemophilum: Mycobacterium haemophilum; MGIT: Mycobacteria Growth Indicator Tube; NIDDM: non-insulin-dependent diabetes mellitus; NPL: no light perception; NTM: Non-tuberculous mycobacteria; PCR: polymerase chain reaction; $\mathrm{PJ}$ : light projection; POAG: primary open angle glaucoma; PPV: pars plana vitrectomy
}

\section{Acknowledgements}

The authors acknowledge Wilawan Sanpan from Research Division, Faculty of Medicine Siriraj Hospital, Mahidol University, Bangkok, Thailand; for preparing the manuscript.

Funding

No funding to declare in this study.

\section{Availability of data and materials}

The data that support the findings of this study are available from the corresponding author $(\mathrm{PC})$ upon reasonable request.

\section{Authors' contributions}

SS, WP, and PC collected medical information and drafted the article. WP and PC did the literature search and wrote the manuscript. NT and SB contributed to the critical review of the manuscript. All authors revised and approved the final version of the manuscript.

\section{Ethics approval and consent to participate}

The study was in accordance with the tenets of the Declaration of Helsinki. As a case report of one unidentifiable patient, the Committee for the Protection of Human Participants in Research of the Faculty of Medicine Siriraj Hospital, Mahidol University does not require the ethics approval.

\section{Consent for publication}

Written informed consent was obtained from the patient for publication of the case report. A copy of the written consent is available for review by the Editor of this journal.

\section{Competing interests}

The authors declare that they have no competing interests.

\section{Publisher's Note}

Springer Nature remains neutral with regard to jurisdictional claims in published maps and institutional affiliations.

Received: 13 November 2017 Accepted: 31 January 2018

Published online: 07 February 2018

\section{References}

1. Runyon EH. Anonymous mycobacteria in pulmonary disease. Med Clin North Am. 1959:43:273-90.

2. Falkinham JO 3rd. Surrounded by mycobacteria: nontuberculous mycobacteria in the human environment. J Appl Microbiol. 2009;107:356-67.

3. Lindeboom JA, van Coppenraet LEB, van Soolingen D, Prins JM, Kuijper EJ. Clinical manifestations, diagnosis, and treatment of mycobacterium haemophilum infections. Clin Microbiol Rev. 2011;24:701-17.

4. Smith S, Taylor GD, Fanning EA. Chronic cutaneous mycobacterium haemophilum infection acquired from coral injury. Clin Infect Dis. 2003; 37:e100-1.

5. Vaerewijck MJ, Huys G, Palomino JC, Swings J, Portaels F. Mycobacteria in drinking water distribution systems: ecology and significance for human health. FEMS Microbiol Rev. 2005;29:911-34.

6. Sompolinsky D, Lagziel A, Naveh D, Yankilevitz T. Mycobacterium haemophilum sp. nov., a new pathogen of humans. Int I Syst Evol Microbiol. 1978:28:67-75.

7. Hirsch R, Miller SM, Kazi S, Cate TR, Reveille JD. Human immunodeficiency virus-associated atypical mycobacterial skeletal infections. Semin Arthritis Rheum. 1996;25:347-56.

8. Kelley CF, Armstrong WS, Eaton ME. Disseminated mycobacterium haemophilum infection. Lancet Infect Dis. 2011;11:571-8.

9. Lindeboom JA, Kuijper EJ, Bruijnesteijn van Coppenraet ES, Prins JM. First case of an oculofacial lesion due to mycobacterium haemophilum infection in an immunocompetent child. Oral Surg Oral Med Oral Pathol Oral Radiol Endod. 2006;101(6):774.

10. Millar MJ, Bulliard C, Balachandran C, Maloof AJ. Mycobacterium hemophilum infection presenting as filamentary keratopathy in an immunocompromised adult. Cornea. 2007;26(6):764.

11. Zuercher B, Waridel F, Monnier P, Cherpillod J. A case of dacryocystitis due to M. Haemophilum. Int J Pediatric Otorhinolaryngology Extra. 2011;6(4):261. 
12. Modi D, Pyatetsky D, Edward DP, et al. Mycobacterium haemophilum: a rare cause of endophthalmitis. Retina. 2007;27:1148-51.

13. Gedde SJ, Scott IU, Tabandeh H, et al. Late endophthalmitis associated with glaucoma drainage implants. Ophthalmology. 2001;108(7):1323.

14. Shah M, Relhan N, Kuriyan AE, et al. Endophthalmitis caused by nontuberculous mycobacterium: clinical features, antimicrobial susceptibilities, and treatment outcomes. Am J Ophthalmol. 2016;168:150-6.

15. Vaziri K, Kishor K, Schwartz SG, et al. Incidence of bleb-associated endophthalmitis in the United States. Clinical Ophthalmology (Auckland, NZ). 2015;9:317-22.

16. Yamamoto T, Sawada A, Mayama C, et al. The 5-year incidence of blebrelated infection and its risk factors after filtering surgeries with adjunctive mitomycin C: collaborative bleb-related infection incidence and treatment study 2. Ophthalmology. 2014;121:1001-6.

17. Dawson DJ, Jennis F. Mycobacteria with a growth requirement for ferric ammonium citrate, identified as mycobacterium haemophilum. J Clin Microbiol. 1980;11(2):190-2.

18. Straus WL, Ostroff SM, Jernigan DB, et al. Clinical and epidemiologic characteristics of mycobacterium haemophilum, an emerging pathogen in immunocompromised patients. Ann Intern Med. 1994;120:118-25.

19. Moorthy RS, Valluri S, Rao NA. Nontuberculous mycobacterial ocular and adnexal infections. Surv Ophthalmol. 2012;57:202-35.

20. Saubolle MA, Kiehn TE, White MH, Rudinsky MF, Armstrong D. Mycobacterium haemophilum: microbiology and expanding clinical and geographic spectra of disease in humans. Clin Microbiol Rev. 1996;9:435-47.

21. White MH, Papadopoulos EB, Small TN, Kiehn TE, Armstrong D. Mycobacterium haemophilum infections in bone marrow transplant recipients. Transplantation. 1995;60:957-60.

22. Fairhurst RM, Kubak BM, Pegues DA, Moriguchi JD, Han KF, Haley JC, et al. Mycobacterium haemophilum infections in heart transplant recipients: case report and review of the literature. Am J Transplant. 2002;2:476-9.

\section{Submit your next manuscript to BioMed Central and we will help you at every step:}

- We accept pre-submission inquiries

- Our selector tool helps you to find the most relevant journal

- We provide round the clock customer support

- Convenient online submission

- Thorough peer review

- Inclusion in PubMed and all major indexing services

- Maximum visibility for your research

Submit your manuscript at www.biomedcentral.com/submit 